\title{
In-band full-duplex medium access control design for heterogeneous wireless LAN
}

\author{
Md. Abdul Alim ${ }^{1,2^{*}}$, Makoto Kobayashi ${ }^{1}$, Shunsuke Saruwatari ${ }^{1}$ and Takashi Watanabe ${ }^{1}$
}

\begin{abstract}
Full-duplex (FD) wireless communication is evolving into a practical technique, and many studies are being conducting in this area, especially regarding the physical layer. However, to exploit FD benefit successfully, efficient medium access control (MAC) protocols are crucial as well as physical layer advances. Numerous FD-MAC protocols have been proposed, but these MAC protocols cannot address all the issues encountered in this area. In addition, many half-duplex (HD) capable devices are present in existing wireless local area networks (WLANs), so there is an urgent need to integrate FD clients and HD clients in the same WLAN. We refer to this type of WLAN as a heterogeneous WLAN (Het-WLAN). In this paper, we propose an FD-MAC for Het-WLAN, which considers all possible types of FD transmissions. Our proposed FD-MAC protocol suppresses inter-user interference. Simulation results demonstrated that a significant throughput gain (about 96\%) could be achieved by using our proposed FD-MAC compared with traditional HD communications. Moreover, our proposed MAC obtained better performance (average throughput gain of about $11 \%$ ) compared with another existing FD-MAC design. In addition, probability analysis suggested that the total probability of FD transmissions increased rapidly as the WLAN approached saturation conditions.
\end{abstract}

Keywords: Full-duplex, MAC protocol, Het-WLAN, FD-MAC

\section{Introduction}

In general, traditional radio transceivers cannot transmit and receive simultaneously using the same frequency band because of self-interference at the receiver end. However, recent technological advances in antenna design and radio frequency interference cancellation techniques can reduce self-interference by up to $110 \mathrm{~dB}$ [1]. Similar studies have also been conducted regarding the physical layer by [2-4]. The latest technologies for self-interference cancellation allow us to transmit and receive signals simultaneously using the same frequency, which is known as in-band full-duplex (IBFD) communication [5]. However, a suitable medium access control (MAC) is crucial to exploit the full advantages of IBFD technology in wireless local area networks (WLANs) because the current IEEE standard MAC protocols do not support IBFD communications. IBFD is one of the techniques with the greatest potential for supporting the huge traffic demands in the near future, and researchers are attracted to IBFD

\footnotetext{
*Correspondence: abdul.alim@ist.osaka-u.ac.jp

${ }^{1}$ Department of Information Networking, Graduate School of Information

Science and Technology, Osaka University, Osaka, Japan

${ }^{2}$ ECE Discipline, Khulna University, Khulna, Bangladesh
}

because it can increase the spectral efficiency without requiring any additional frequency resources [5]. IBFD can also double the ergodic capacity of a multiple-input multiple-output system $[1,3]$.

IBFD wireless communication can be categorized as bidirectional FD (BFD), three node FD (TNFD), or relay FD (RFD) [5, 6]. BFD and TNFD are illustrated in Fig. 1. Two nodes comprising the primary transmitter (PT) and primary receiver (PR) transmit to and receive from each other simultaneously (Fig. 1a) in BFD. TNFD can be described as destination-based TNFD or source-based TNFD. In destination-based TNFD, PT transmits a signal to PR and PR also transmits a signal to another secondary receiver (SR) while receiving data from PT (Fig. 1b). In this case, PR also acts as a secondary transmitter (ST). In source-based TNFD, PT transmits data to PR and ST also transmits data to PT (Fig. 1c). In this case, PT also acts as a SR. However, in RFD, a user terminal sends data to the destination using a relaying node [5]. In this study, we consider three modes of transmission, i.e., half-duplex (HD), $\mathrm{BFD}$, and TNFD. 


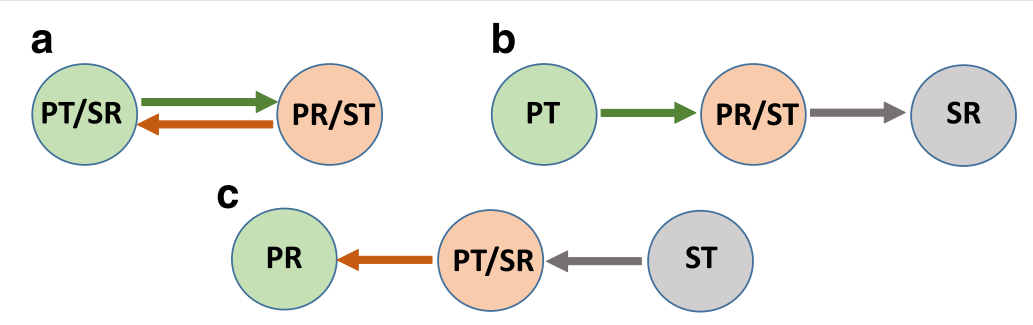

Fig. 1 a BFD, b destination-based TNFD, and c source-based TNFD

All of the user terminals or nodes in existing WLANs are traditional HD capable. Therefore, it is not possible to replace all of these HD nodes (HDNs) with FD nodes (FDNs) overnight, so it is necessary to incorporate FDNs in existing WLANs in a manner that allows HDNs and FDNs to operate simultaneously. A WLAN that comprises HDNs and FDNs is referred to as a heterogeneous WLAN (Het-WLAN) because it has different types of clients or nodes.

In this paper, we propose an FD-MAC for Het-WLAN, which we call HFD-MAC. The basic structure of HetWLAN is shown in Fig. 2, where the access point (AP) is FD capable (FD-AP) and some FDNs are present as well as HDNs. Moreover, Het-WLAN is important because HDNs have some advantages compared with FDNs. For example, FDNs are more expensive and they consume more power. Therefore, some people may not like FD devices, and they will prefer to choose HD devices to meet their daily needs. Therefore, a suitable FD-MAC design is required for this type of Het-WLAN.
The symmetric length of the uplink and downlink data traffic is considered in the design of our FD-MAC protocol. This type of traffic is very common in different areas, such as cellular mobile communications, distant health monitoring, online video games, peer-to-peer (P2P) TV, P2P file sharing, and video conferencing [7]. Moreover, IBFD wireless communications should be designed in such a way, where the symmetric traffic length plays a vital role. Otherwise, the link (downlink or uplink) that finishes its data transmission earlier will be idle for the remainder of the time of that round.

In this HFD-MAC protocol, all possible types of FD transmission are considered. To the best of our knowledge, this is a novel research that describes the FD-MAC for this kind of Het-WLAN, which describes all possible transmissions. The main contributions of this research are as follows.

- An FD-MAC is proposed for a Het-WLAN that contains FDNs and HDNs.

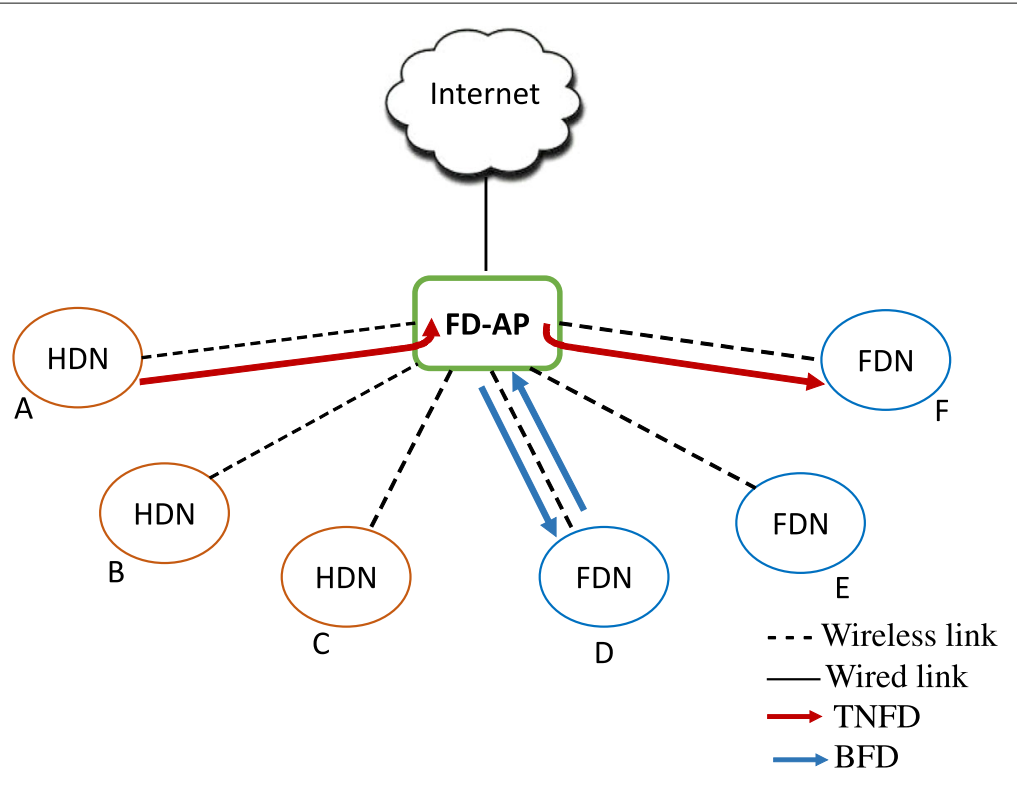

Fig. 2 Structure of a Het-WLAN 
- The proposed FD-MAC considers all possible types of FD transmissions in this Het-WLAN.

- We compared performance of our proposed FD-MAC with traditional HD communications and another existing FD-MAC protocol.

- A comprehensive probability analysis was performed for the proposed HFD-MAC.

The remainder of this paper is organized as follows. In Section 2, we provide a brief description of related research in this area. In Section 3, we present the problem statement regarding FD-MAC. In Section 4, we explain the proposed MAC design. In Section 5, we give a brief description of the method for combating inter-user interference. In Section 6, we present the mathematical analysis. The results and performance analysis are given in Section 7. In Section 8, we give our conclusions.

\section{Related works}

Many studies in the area of IBFD wireless communication have aimed to minimize self-interference, which are related to the physical layer. For example, a single antenna was used to support IBFD WiFi radio by [1], where both analog and digital self-interference cancelation techniques were used. Various techniques have been proposed to reduce self-interference, which are related to advances in IBFD wireless communication in the physical layer $[2,4,8-11]$.

Although several MAC designs have been proposed for WLANs using IBFD, they do not consider all possible types of IBFD transmissions. In addition, some were proposed for distributed wireless networks or ad hoc networks, where all the clients were treated as FDNs $[12,13]$. An FD multi-channel MAC (FD-MMAC) was proposed to mitigate multi-channel hidden terminal (HT) problems, which targeted for eliminating controlling signals [12]. During data transmission between two nodes, the receiver transmits a beacon packet back-to-back until the data transmission is complete. This beacon packet does not contain any user data. Hence, in terms of user data communications, the FD capability is not fully utilized in FD-MMAC. In addition, TNFD communication is not possible because PR always needs to transmit beacon packets while receiving data from PT. Another MAC protocol was proposed by [13] for IBFD ad-hoc networks, where all the clients were considered as FDNs. The interuser interference is not considered during TNFD communications, and thus, TNFD communications will be affected greatly by inter-user interference problems. The MAC design proposed by [14] can be used in both ad hoc and infrastructure-based WLANs. In this design, all the nodes are considered to be FD capable. Moreover, in the case of source-based TNFD transmissions, collisions will occur if more than two clients select the same sub-carrier and AP also selects that sub-carrier. Furthermore, this MAC does not include a procedure to allow the network allocation vector (NAV) to update the timing in different nodes.

An infrastructure-based MAC protocol for IBFD wireless communications was proposed that includes a shared random back-off mechanism [15], where all the nodes are treated as FDNs and the nodes can switch to the HD or FD mode based on the traffic availability. According to this MAC, all the nodes first participate in the normal contention period, before HD transmission occurs. Subsequently, any FD transmission (BFD or TNFD) can be performed after the shared random back-off period. Hence, a HD transmission should be performed earlier before initiating an FD communication. Moreover, the method proposed by [15] restricts TNFD transmission to this case only, where AP wins in the shared random backoff period. A power-controlled MAC (PoCMAC) was proposed recently for IBFD WiFi networks [16]. This MAC utilizes a contention-based receiver selection scheme to mitigate inter-user interference during FD transmissions, but only FD-AP and HD clients are considered in the network. The AP cannot initiate any transmissions in PoCMAC. Another FD-MAC design was proposed for a WiFi network, where both HD and FD clients are available [17]. However, the authors limit FD communications when a mobile node and AP have packets for each other.

An FD-MAC was proposed for a WLAN by [18], where AP is FD capable and all the clients are traditionally HD capable. In this method, AP and all the clients need to calculate and update the signal-to-interference ratio map continuously, which may increase the computational load as well as the complexity. Transmissions always need to be initiated by clients to establish IBFD communications. If AP wins in the contention period, IBFD communication cannot be established for two reasons: the clients do not support IBFD transmissions or the clients cannot make decisions about which can start the TNFD transmission. Another AP-based MAC protocol was proposed by [19], where all of the clients and AP are FD capable. This protocol has a polling-based MAC design, where AP always needs to initiate the transmission. However, the control frame formats were not described clearly in this MAC design.

Other studies have considered different issues on IBFD communications, such as relay transmissions or power control-based FD [20-22], but they did not consider the MAC design.

\section{Problem statement}

The hidden and exposed terminal problems are not so significant when the network is considered to be an AP-based WLAN and communication is performed using a request-to-send (RTS) and clear-to-send (CTS) 
handshake mechanism. This is because all the clients in a WLAN can update their NAVs by using the control frames transmitted by AP.

However, inter-user interference is a major issue in FDWLAN. The inter-user interference problem is illustrated in Fig. 3. As shown in the figure, node A sends an RTS to the AP (FD capable) first and, then, the AP makes a decision to send data to node $\mathrm{D}$ while also receiving data from node $\mathrm{A}$. If nodes $\mathrm{A}$ and $\mathrm{D}$ are close to each other, the packets from node A will interfere with the packets sent by the AP to node D, thereby producing inter-user interference problems. Node A and the AP are regarded as PT and PR, respectively. However, node D and the AP are treated as SR and ST, respectively. Therefore, AP acts as both PR and ST. Without taking appropriate steps to mitigate this inter-user interference, IBFD communications cannot be performed perfectly.

\section{Proposed MAC protocol: HFD-MAC}

\subsection{New control frames}

A number of control frames are used in HFD-MAC proto$\mathrm{col}$, where some are the same as those in the IEEE 802.11 standards, such as RTS, CTS, and acknowledgement (ACK). However, some new control frames are introduced in this HFD-MAC, as shown in Fig. 4, e.g., RTS with duplexing indicator (RTSD) and CTS with duplexing indicator (CTSD). The duplexing indicator (DI) is a two-bit value, which is appended to the normal RTS and CTS to obtain RTSD and CTSD, respectively, thereby facilitating FD transmissions. A description of the DI value is given in Table 1. FDNs always use RTSD and CTSD, whereas HDNs use RTS and CTS. In addition, AP uses RTSD and CTSD to communicate with FDNs. AP also uses RTS and CTS, if it communicates with HDNs. Another two control frames called new-CTS (NCTS) and notification with DI value (NDI) are transmitted only by AP. The transmission (Tx) capabilities of the control frames are summarized in Table 2, where "circles" and "crosses" indicate capability and incapability, respectively.

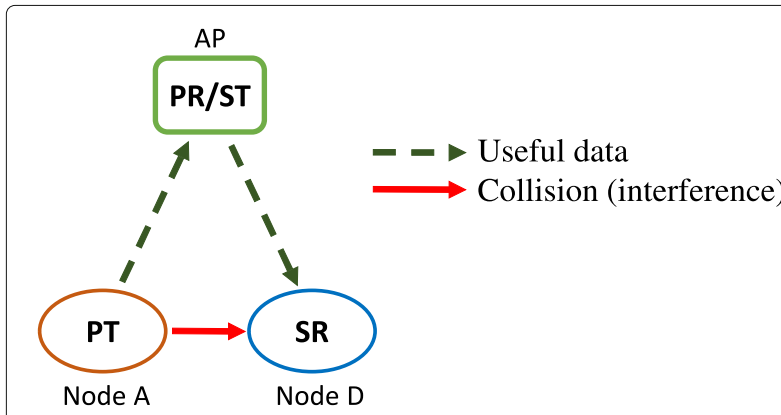

Fig. 3 Inter-user interference in FD-WLAN

\subsection{Possible FD transmission in Het-WLAN}

HFD-MAC is proposed for a Het-WLAN, which comprises an FD-AP, FDNs, and HDNs (Fig. 2). All possible cases of FD transmissions are summarized in the following two main cases.

\section{Case 1: TNFD communications}

(i) AP initiates the transmission

(ii) Any HDN initiates the transmission

(iii) Any FDN initiates the transmission

\section{Case 2: BFD communications}

In HFD-MAC, the TNFD communication is performed between the AP and two other nodes, which can be an HDN or FDN. The BFD communication is performed between the AP and an FDN when they have data for each other. However, HD transmissions may also occur in the proposed MAC.

\subsection{Description of the proposed HFD-MAC}

The working principle of this HFD-MAC is based on the distributed coordination function (DCF) in IEEE 802.11. This protocol uses CSMA/CA and a back-off mechanism to access the channel and to avoid collisions. For simplicity, the DCF interframe space (DIFS) time and back-off time are not shown in the figures, but the short interframe space (SIFS) time is shown by "S" in all of the figures. In this MAC, a node ignores collisions or the reception of erroneous packets during the NAV period. Moreover, it is assumed that FDNs can perform self-interference cancelation perfectly.

According to the IEEE 802.11 standard, a number of management frames are exchanged between the AP and nodes when nodes or devices join the WLAN [23]. For example, after exchanging the prob request and response, the AP grants access to the device if the device can satisfy the authentication and association procedure. Some optional bits in these management frames can be used by the nodes to indicate their FD capabilities. For example, during the joining process, a node moves to the association phase after the authentication procedure and an association request is sent to the AP. The node can indicate its FD capability using this association request because several optional bits are available. Therefore, the AP can determine which MAC address has an FD capability and which has an HD capability. Hence, the AP sends an RTSD to the FDN and an RTS to the HDN. In addition, RTS and RTSD are transmitted by HDNs and FDNs, respectively.

The description of this MAC protocol is given below for the cases mentioned above. 


\begin{tabular}{|c|c|c|c|c|c|c|}
\hline $\begin{array}{c}\text { NCTS Frame } \\
20 \text { Bytes }\end{array}$ & $\begin{array}{c}\text { Frame } \\
\text { Control (2) }\end{array}$ & $\begin{array}{c}\text { Duration } \\
\text { (2) }\end{array}$ & $\begin{array}{l}\text { FRA } \\
(6)\end{array}$ & $\begin{array}{l}\text { SRA } \\
\text { (6) }\end{array}$ & $\begin{array}{r}\text { CRC } \\
(4)\end{array}$ & \\
\hline $\begin{array}{l}\text { RTSD Frame } \\
20.25 \text { Bytes }\end{array}$ & $\begin{array}{c}\text { Frame } \\
\text { Control (2) }\end{array}$ & $\begin{array}{c}\text { Duration } \\
\text { (2) }\end{array}$ & $\begin{array}{l}\text { RA } \\
\text { (6) }\end{array}$ & $\begin{array}{l}\text { TA } \\
\text { (6) }\end{array}$ & $\begin{array}{c}\mathrm{DI} \\
\text { (2 bits) }\end{array}$ & $\begin{array}{r}\text { CRC } \\
\text { (4) }\end{array}$ \\
\hline $\begin{array}{l}\text { CTSD Frame } \\
14.25 \text { Bytes }\end{array}$ & $\begin{array}{c}\text { Frame } \\
\text { Control (2) }\end{array}$ & $\begin{array}{c}\text { Duration } \\
\text { (2) }\end{array}$ & $\begin{array}{l}\text { RA } \\
(6)\end{array}$ & $\begin{array}{c}\text { DI } \\
\text { (2 bits) }\end{array}$ & $\begin{array}{r}\text { CRC } \\
\text { (4) }\end{array}$ & \\
\hline $\begin{array}{c}\text { NDI } \\
14.25 \text { Bytes }\end{array}$ & $\begin{array}{c}\text { Frame } \\
\text { Control (2) }\end{array}$ & $\begin{array}{c}\text { Duration } \\
\text { (2) }\end{array}$ & $\begin{array}{c}\text { AP address } \\
\text { (6) }\end{array}$ & $\begin{array}{r}D \\
(2 b\end{array}$ & & $\begin{array}{l}\text { CRC } \\
\text { (4) }\end{array}$ \\
\hline & \multicolumn{6}{|c|}{$\begin{array}{l}\text { RA: Receiver Address } \\
\text { TA: Transmitter Address } \\
\text { FRA: First Receiver Address } \\
\text { SRA: Second Receiver Address } \\
\quad \text { : Newly added bits }\end{array}$} \\
\hline
\end{tabular}

Fig. 4 Control frame format

\subsubsection{Case 1: TNFD communications}

\section{(i) AP initiates the transmission}

The AP initiates the transmission if it wins the contention period. If the AP has data for a HDN, it sends an RTS to the corresponding HDN. For example, the AP sends an RTS to a HDN (A) and A sends a CTS to AP, as shown in Fig. 5. If another node (HDN/FDN) then wants to send data to AP, it needs to satisfy secondary data transmission condition (SDTC)-1: "After receiving RTS from AP, ST cannot hear the CTS from A." AP sends the RTS, so all the nodes know that AP can receive data from another node. Hence, after hearing an RTS from AP, all the other nodes (HDN and/or FDN) that want to send data to AP must wait for a time (SIFS+CTS). During this time, the nodes that have data to send AP and that cannot hear A's CTS stop their NAVs and start their self-timers. The timer sets a random time within a maximum limit and the timer decreases its value. The node with the timer that stops first starts to send data to AP. As shown in Fig. 5, B's timer stops first and it starts to send data to AP. Before sending data to AP, the node (B) senses whether the channel is busy or not. Thus, other nodes (which started their self-timers) will stop their timers sequentially and resume their NAVs. Immediately after receiving data from ST, AP starts to transmit data to $\mathrm{A}$. The corresponding receivers transmit ACKs after the data transmission.

Table 1 Description of duplexing indicator

\begin{tabular}{ll}
\hline Value of DI & Meaning of DI \\
\hline 00 & Not used \\
01 & Sender can Rx only \\
10 & Sender can Tx only \\
11 & Sender can Tx and Rx simultaneously \\
\hline
\end{tabular}

In addition, AP sends an RTSD to an FDN if it has data for the FDN. AP always sends an RTSD to an FDN with a DI value of 11 . Hence, all nodes are informed that AP wants to transmit and receive simultaneously. Suppose that AP has sent an RTSD to a FDN (E) with a DI value of 11 and $E$ has no data to send AP (Fig. 6). In this case, E will send a CTSD with a DI value of 01 , which means that $E$ can only receive but cannot transmit because it has no data for AP. AP can receive data from another node, so it transmits an NDI, where the DI value is 10 . This means that AP will only transmit, and thus, other nodes can send data to AP. Based on this NDI, other nodes will be informed that AP can only transmit because it transmitted an RTSD earlier. Therefore, the nodes that want to send data to AP need to satisfy condition SDTC-2: "After hearing an RTSD from AP, ST cannot hear a CTSD from E but it can hear an NDI." The nodes that satisfy SDTC-2 and that want to send data to AP will stop their NAVs and start their selftimers. Suppose that B and C have data to send and they satisfy SDTC-2. As shown in Fig. 6, they start their selftimers after stopping their NAVs. If we suppose that B's timer expires first, then it will send data after sensing the channel as idle. Immediately after receiving data from B,

Table 2 Control frames' transmission capability

\begin{tabular}{lccc}
\hline \multirow{2}{*}{ Control frame } & \multicolumn{3}{c}{ Tx capability } \\
\cline { 2 - 4 } & AP & FDN & HDN \\
\hline RTSD & 0 & $\bigcirc$ & $\times$ \\
CTSD & $\bigcirc$ & $\bigcirc$ & $\times$ \\
RTS & $\bigcirc$ & $\times$ & $\bigcirc$ \\
CTS & $\bigcirc$ & $\times$ & $\bigcirc$ \\
NCTS & $\bigcirc$ & $\times$ & $\times$ \\
NDI & 0 & $\times$ & $\times$ \\
\hline
\end{tabular}




\begin{tabular}{|c|c|c|c|c|c|c|c|}
\hline \multicolumn{2}{|l|}{ A } & $S$ & CTS & & & S & ACK \\
\hline AP & RTS & & & & Data to $A$ & $\mathrm{~S}$ & ACK \\
\hline \multicolumn{2}{|l|}{ C } & NA & $\mathrm{V}$ (RTS) & Timer & Data to AP & & \\
\hline \multicolumn{2}{|l|}{$\mathrm{E}$} & \multicolumn{2}{|c|}{ NAV(RTS) } & Timer & \multicolumn{2}{|c|}{ Restart NAV(RTS) } & \\
\hline \multicolumn{2}{|c|}{ Others } & \multicolumn{6}{|c|}{ NAV (RTS) } \\
\hline \multicolumn{2}{|c|}{$(B, D, F)$} & & & & NAV (CTS) & & \\
\hline
\end{tabular}

Fig. 5 AP initiates the transmission to HDN

AP starts transmission to $E$. In addition, $C$ will stop the timer and resume its NAV. All of the other nodes (A, D, and F) will update their NAVs using the NDI and the ACKs are transmitted accordingly.

(ii) Any HDN initiates the transmission

An HDN initiates the transmission by sending an RTS to AP if the HDN wins the contention period for the channel access. According to Fig. 7, after receiving an RTS from a HDN (where the HDN is node A), AP may have data to send to another node, which may be another HDN or an FDN. In this case, if we suppose that AP wants to send data to an SR (B), then AP will mention the address of $B$ in the SR address (SRA) of its reply in a new-CTS (NCTS), as shown in Fig. 4. The NCTS will inform both $A$ and $B$ about their data exchange information. Node $A$ updates its NAV after receiving the NCTS. After receiving the NCTS from AP, B replies by sending another CTS to AP based on SDTC-3: "SR can only hear the NCTS but not the RTS from A." If SDTC-3 is satisfied by B, then it sends a CTS to AP. The data transmission then occurs as shown in Fig. 7. After the data transmission, ACKs are sent simultaneously. However, if AP does not receive the CTS from SR, it receives data from A after finishing the SIFS and CTS time allocated to B. Other nodes update their NAV times according to the time defined by the control frames.

(iii) Any FDN initiates the transmission

After winning in the contention period, an FDN initiates a transmission by sending an RTSD to AP with a DI value of 11. AP does not have data to send the corresponding FDN, and thus, it is a TNFD communication. However, AP has data for another node (may be another FDN or any HDN). Hence, AP transmits an NCTS. The subsequent procedure is the same as that described in the previous subsection for case 1 (ii). Therefore, sending an NCTS after receiving an RTSD from AP indicates that AP has data for another node.

\subsubsection{Case 2: BFD communications}

BFD transmission occurs between AP and one FDN when they have data for each other. In this case, either AP or an FDN wins in the contention period and sends an RTSD.

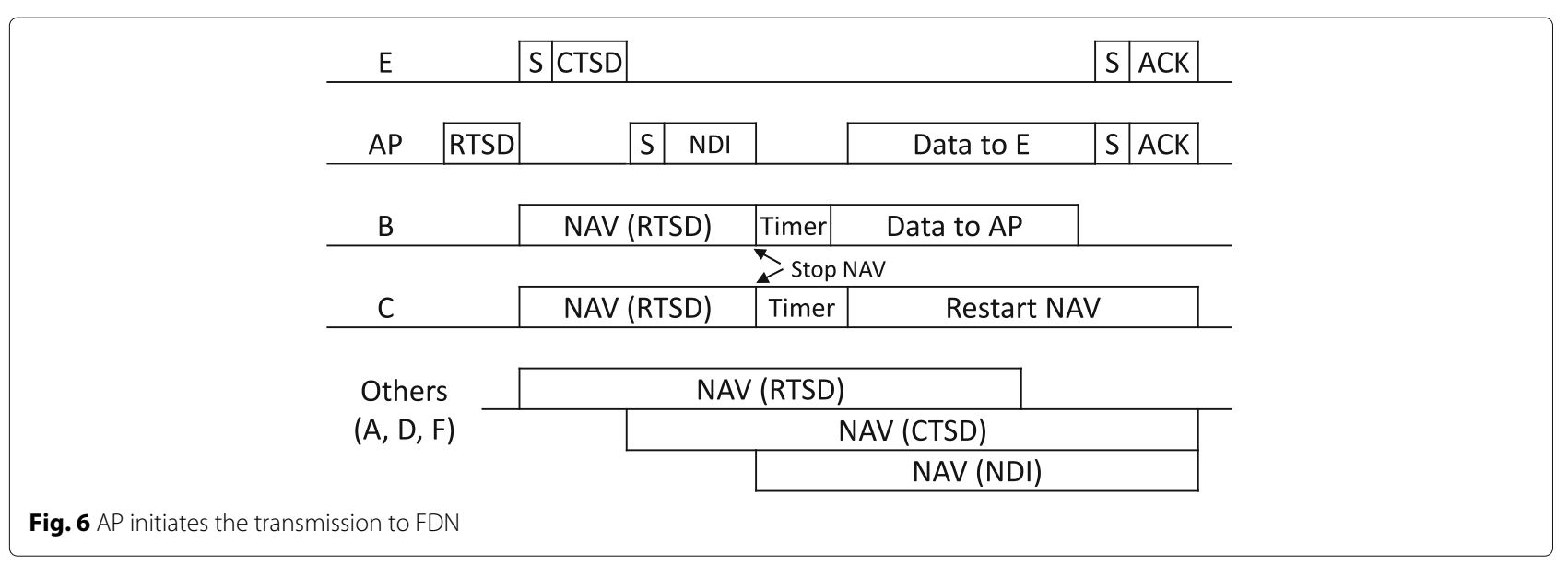




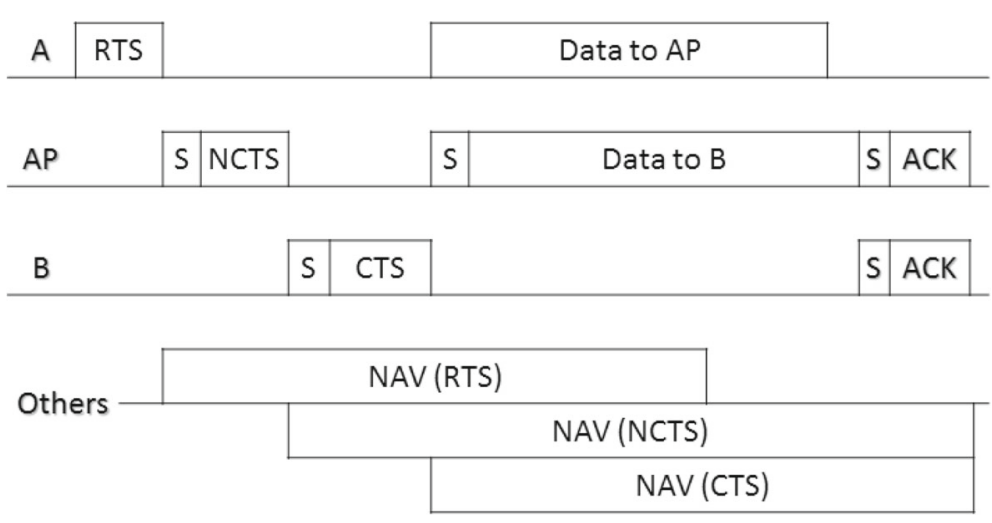

Fig. 7 A HDN initiates the transmission

For example, AP sends an RTSD with a DI value of 11 to an FDN. The corresponding FDN also sends a CTSD with a DI value of 11 because the FDN has data for AP. BFD transmission then occurs.

It should be noted that an RTSD is always sent with a DI value of 11 . The corresponding data transmission may be BFD, TNFD, or HD, which depends on the reply of the CTSD and other control frames.

\section{Combating inter-user interference}

Our proposed MAC for Het-WLAN suppresses inter-user interference using the following three SDTCs.

- SDTC-1: "An ST can hear an RTS from AP but cannot hear the corresponding CTS from PR."

- SDTC-2: "An ST can hear an RTSD from AP and cannot hear the corresponding CTSD from PR, but can hear the NDI from AP."

- SDTC-3: "An SR cannot hear an RTS/RTSD from the PT but can hear the corresponding NCTS from AP."

SDTC-1 is applied to TNFD communication where AP initiates the transmission to a HDN. Similarly, SDTC-2 is applied in the case of TNFD where AP initiates the transmission to an FDN. Thus, after initiating the transmission by the AP to a FDN, if an ST wants to send data to the AP, the ST needs to satisfy SDTC-2, i.e., if the ST has data to send to AP and satisfies SDTC-2, it stops its NAV and starts the self-timer, and the corresponding procedures occur for the TNFD communication, as described in the previous section. In addition, SDTC-3 is used in the case where an HDN/FDN initiates the TNFD communication. These FD-MAC mechanisms prevent inter-user interference during TNFD communications.

For example, as shown in Fig. 8, node A (PT) sends an RTS to AP, which is heard by node $C$, but it is not heard by B. Next, AP sends an NCTS to A and B because AP wants to send data to $B$, which is now an SR. In this case, $B$ will send a CTS because it has satisfied SDTC-3. If B cannot satisfy the condition, B will not send the CTS and it becomes an HD transmission. If the conditions are not maintained by SRs and STs, inter-user interference occurs among the user terminals during TNFD communications. Thus, TNFD communications will sometimes fail due to data collision.

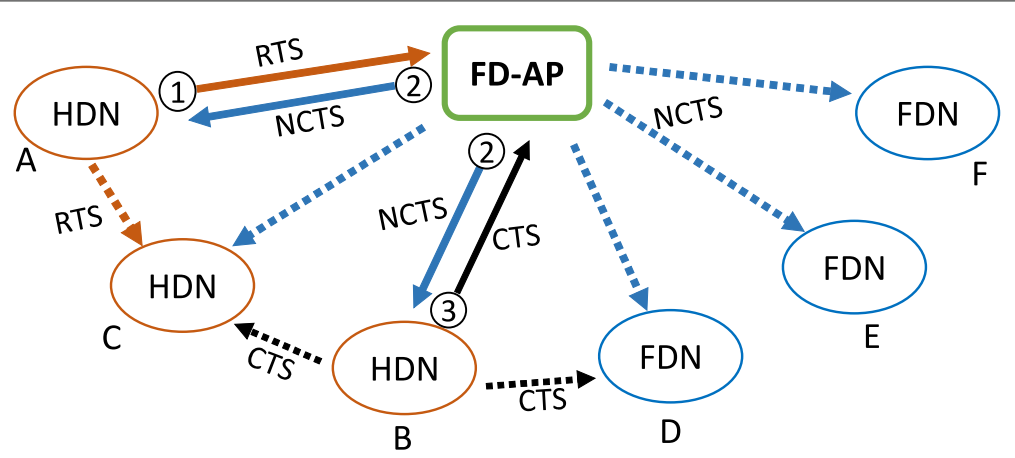

Solid line: useful data, Dotted line: unwanted data

Fig. 8 SDTC-3: this is satisfied by $B$; however, it is not satisfied by $C$ 


\section{Mathematical analysis}

In this section, we derive the probability equations for different transmissions. The probability equations for different types of communication (BFD, TNFD, and HD) are derived by using the packet arrival rate (PAR) at AP for the nodes and the packet generation rate (PGR) by clients or nodes. It is assumed that a percentage $(\gamma)$ of total nodes act as HTs. Satisfying SDTC is a key factor that affects the performance of TNFD communications, and it depends on the number of HTs. Therefore, we considered HTs when deriving the equations. The descriptions of different symbols and variables in the equations are given in Table 3. All packet arrivals are assumed to follow a Poisson process, and we assume that the system has the characteristics of the $\mathrm{M} / \mathrm{M} / 1$ system model.

The PAR at AP from the Internet to all clients is

$$
\lambda_{A P}=m \lambda_{\mathrm{APH}}+n \lambda_{\mathrm{APF}}
$$

The total PAR at AP from the Internet and clients is

$$
\lambda_{\text {Total }}=\lambda_{\mathrm{AP}}+m \lambda_{\mathrm{H}}+n \lambda_{\mathrm{F}}
$$

If one packet arrives at AP from the Internet, the conditional probability of that packet arriving at AP for an FDN is $\frac{n \lambda_{\text {APF }}}{\lambda_{\text {Total }}}$ and the conditional probability of that packet arriving for an $\mathrm{HDN}$ is $\frac{m \lambda_{\mathrm{APH}}}{\lambda_{\text {Total }}}$. Similarly, if a packet arrives at AP from a node, the conditional probability of that packet arriving from an FDN can be defined as $\frac{n \lambda_{\mathrm{F}}}{\lambda_{\text {Total }}}$ and

Table 3 Declaration of variables

\begin{tabular}{ll}
\hline Variables & Explanation \\
\hline$m$ & Number of HDNs \\
$n$ & Number of FDNs \\
$\gamma$ & Percentage of total nodes as hidden terminals \\
$\lambda_{\text {AP }}$ & Packet arrival rate at AP for all clients (packets/s) \\
$\lambda_{\text {APH }}$ & Packet arrival rate at AP for each HDN (packets/s) \\
$\lambda_{\text {APF }}$ & Packet arrival rate at AP for each FDN (packets/s) \\
$\lambda_{H}$ & Packet generating rate by each HDN for AP (packets/s) \\
$\lambda_{F}$ & Packet generating rate by each FDN for AP (packets/s) \\
$P_{\text {BFD }}$ & Probability of bidirectional FD communication \\
$P_{\text {TNFD }}$ & Probability of TNFD communication \\
$P_{\text {HD }}$ & Probability of HD communication \\
$T_{\text {RTS }}$ & Time for RTS \\
$T_{\text {CTS }}$ & Time for CTS \\
$T_{\text {RTSD }}$ & Time for RTSD \\
$T_{\text {CTSD }}$ & Time for CTSD \\
$T_{\text {SIFS }}$ & Time for SIFS \\
$T_{\text {NDI }}$ & Time for NDI \\
\hline
\end{tabular}

the conditional probability of that packet arriving from an HDN is $\frac{m \lambda_{H}}{\lambda_{\text {Total }}}$. During any data transmission, the packets may still arrive at nodes or AP. However, the nodes differ to start the transmission because the channel is busy. Therefore, we need to know the average waiting time $\left(T_{w}\right)$ for a packet in the queue, which can be derived as follows:

$$
\begin{aligned}
T_{w} & =\frac{\text { arrivalrate }}{\text { servicerate } \times(\text { servicerate }- \text { arrivalrate })} \\
& =\frac{\lambda_{\text {Total }}}{\mu\left(\mu-\lambda_{\text {Total }}\right)}
\end{aligned}
$$

where $\mu$ is the average service rate of AP.

The probability analysis is described in the following subsections.

\subsection{BFD communications}

To derive the probability of bidirectional FD communications $\left(P_{\mathrm{BFD}}\right)$, we present Lemmas 1 and 2.

Lemma 1 After sending an RTSD to an FDN by the AP, $B F D$ will occur only if the corresponding FDN also has data to send to the AP.

Proof To calculate the probability of BFD occurring when AP initiates the transmission to a FDN, we need to calculate the following two probabilities. (a) The conditional probability that the packet arriving at AP is for an FDN, which is $\frac{n \lambda_{\mathrm{APF}}}{\lambda_{\text {Total }}}$. (b) The probability that the corresponding FDN also has a packet to send to the AP within the time $T_{1}$, where $T_{1}=T_{\mathrm{RTSD}}+T_{\mathrm{SIFS}}+T_{w}$. The probability that the FDN has at least one packet to send to AP within time $T_{1}$ is $\left(1-e^{-\lambda_{\mathrm{F}} T_{1}}\right)$. By multiplying (a) and (b), we can calculate the probability of BFD occurring in this case.

Lemma 2 After sending an RTSD to the AP by an FDN, $B F D$ will occur only if the AP also has data to send to the corresponding FDN.

Proof To calculate the probability of BFD occurring when an FDN initiates the transmission, we need to calculate the following two probabilities. (a) The conditional probability that the packet arriving at AP is sent by an FDN, which is $\frac{n \lambda_{F}}{\lambda_{\text {Total }}}$. (b) The probability that AP also has a packet to send to FDN within the time $T_{1}$. The probability that AP has at least one packet to send to the corresponding FDN within time $T_{1}$ is $\left(1-e^{-\lambda_{\mathrm{APF}} T_{1}}\right)$. By mltiplying (a) and (b), we can calculate the probability of BFD occurring in this case. 
By using Lemmas 1 and 2, we can derive an equation to calculate the probability of BFD occurring $\left(P_{\mathrm{BFD}}\right)$, as follows.

$$
\begin{aligned}
P_{\mathrm{BFD}}= & \frac{n \lambda_{\mathrm{APF}}}{\lambda_{\text {Total }}}\left(1-e^{-\lambda_{\mathrm{F}} T_{1}}\right) \\
& +\frac{n \lambda_{\mathrm{F}}}{\lambda_{\text {Total }}}\left(1-e^{-\lambda_{\mathrm{APF}} T_{1}}\right)
\end{aligned}
$$

\subsection{TNFD communications}

To derive the probability of the TNFD communication $\left(P_{\mathrm{TNFD}}\right)$, we present Lemmas 3-6 as follows.

Lemma 3 After initiating the transmission by a HDN to the AP, TNFD communications will occur only if AP also has data to send to any other node that cannot hear the RTS from the HDN.

Proof To calculate the probability of TNFD occurring in the case when an HDN initiates the communication, we need to calculate the following two probabilities. (a) The conditional probability that the packet arriving at AP is sent by an HDN, which is $\frac{m \lambda_{H}}{\lambda_{\text {Total }}}$. (b) The probability that $\mathrm{AP}$ also has a packet to send to any other node that cannot hear the RTS from the HDN within time $T_{2}$, where $T_{2}=$ $T_{\mathrm{RTS}}+T_{\mathrm{SIFS}}+T_{w}$. The probability that AP has at least one packet for one of the hidden nodes within time $T_{2}$ is $\left(1-e^{-\left\{(m-1) \lambda_{\mathrm{APH}}+n \lambda_{\mathrm{APF}}\right\} T_{2}}\right) \gamma$. By multiplying (a) and (b), we can calculate the probability of TNFD occurring in this case.

Lemma 4 After initiating the transmission by a FDN to the AP, TNFD communications will occur only if (i) the AP has no data for the corresponding FDN and (ii) the AP has data for another node that cannot hear the RTSD from the FDN.

Proof To calculate the probability of TNFD occurring in this case, we need to calculate the following three probabilities. (a) The conditional probability that an FDN has a packet to send to AP, which is $\frac{n \lambda_{\mathrm{F}}}{\lambda_{\text {Total }}}$. (b) The probability that AP has no data to send to the corresponding FDN within time $T_{1}$, which is $\left(e^{-\lambda_{\mathrm{APF}} T_{1}}\right)$. (c) The probability that the AP has a packet for another node that cannot hear the RTSD from the FDN within time $T_{1}$, which is $\left(1-e^{-\left\{m \lambda_{\mathrm{APH}}+(n-1) \lambda_{\mathrm{APF}}\right\} T_{1}}\right) \gamma$. We can calculate the probability of TNFD communications occurring in this case by multiplying (a), (b), and (c).

Lemma 5 After initiating the transmission by the AP to a HDN, TNFD communications will occur only if another node that cannot hear the RTS has data to send to AP.
Proof To calculate this probability, we need to calculate the following two probabilities. (a) The conditional probability that the AP has a packet to send a HDN, which is $\frac{m \lambda_{\text {APH }}}{\lambda_{\text {Total }}}$. (b) The probability that any other node that cannot hear the RTS has packets for the AP within time $T_{3}$, where, $T_{3}=T_{\mathrm{RTS}}+T_{\mathrm{SIFS}}+T_{\mathrm{CTS}}+T_{w}$. This probability can be written as $T_{3}$ is $\left(1-e^{-\left\{(m-1) \lambda_{\mathrm{H}}+n \lambda_{\mathrm{F}}\right\} \gamma T_{3}}\right)$. By multiplying (a) and (b), we can calculate the probability of TNFD occurring in this case.

Lemma 6 After initiating the transmission by the AP to a FDN, TNFD will occur only if (i) the corresponding FDN has no data to send to the AP and (ii) any other node that cannot hear the RTSD has data for the AP.

Proof To calculate the probability of TNFD communications occurring in this case, we need to calculate the following three probabilities. (a) The conditional probability that the AP has a packet to send to an FDN, which is $\frac{n \lambda_{\mathrm{APF}}}{\lambda_{\mathrm{Total}}}$. (b) The probability that the corresponding FDN has no data to send to the AP within time $T_{1}$, which is $\left(e^{-\lambda_{\mathrm{F}} T_{1}}\right)$. (c) The probability that any other node that cannot hear the RTSD has data for the AP within time $T_{4}$, where $T_{4}=T_{\mathrm{RTSD}}+2 T_{\mathrm{SIFS}}+T_{\mathrm{CTSD}}+T_{\mathrm{NDI}}+T_{w}$. This probability is calculated as $\left(1-e^{-\left\{m \lambda_{\mathrm{H}}+(n-1) \lambda_{\mathrm{F}}\right\} \gamma T_{4}}\right)$. We can calculate the probability of TNFD occurring in this case by multiplying (a), (b), and (c).

By using Lemmas 3-6, we can derive an equation for calculating the probability of TNFD occurring $\left(P_{\text {TNFD }}\right)$ :

$$
\begin{aligned}
P_{\mathrm{TNFD}}= & \frac{m \lambda_{\mathrm{H}}}{\lambda_{\mathrm{Total}}}\left(1-e^{-\left\{(m-1) \lambda_{\mathrm{APH}}+n \lambda_{\mathrm{APF}}\right\} T_{2}}\right) \gamma \\
& +\frac{n \lambda_{\mathrm{F}}}{\lambda_{\mathrm{Total}}}\left(e^{-\lambda_{\mathrm{APF}} T_{1}}\right)\left(1-e^{-\left\{m \lambda_{\mathrm{APH}}+(n-1) \lambda_{\mathrm{APF}}\right\} T_{1}}\right) \gamma \\
& +\frac{m \lambda_{\mathrm{APH}}}{\lambda_{\text {Total }}}\left(1-e^{-\left\{(m-1) \lambda_{\mathrm{H}}+n \lambda_{\mathrm{F}}\right\} \gamma T_{3}}\right) \\
& +\frac{n \lambda_{\mathrm{APF}}}{\lambda_{\text {Total }}}\left(e^{-\lambda_{\mathrm{F}} T_{1}}\right)\left(1-e^{-\left\{m \lambda_{\mathrm{H}}+(n-1) \lambda_{\mathrm{F}}\right\} \gamma T_{4}}\right)
\end{aligned}
$$

\subsection{HD communications}

To derive the probability of HD communication $\left(P_{\mathrm{HD}}\right)$, Lemmas 7-10 are presented as follows.

Lemma 7 After initiating the transmission by a HDN, $H D$ communications will occur if (i) AP has no data to send any other node or (ii) AP has data to send to another node that is exposed to the corresponding HDN. Because although the AP has data for the nodes that are exposed to the corresponding HDN, the SDTC-3 cannot be satisfied, and thus, a HD communication will occur. 
Proof To calculate the probability of HD communications occurring in this case, we need to calculate the following probabilities. (a) The conditional probability that the HDN initiates a transmission and the probability that the AP has no data to send to other nodes within time $T_{2}$, which is calculated as $\frac{m \lambda_{\mathrm{H}}}{\lambda_{\text {Total }}}\left(e^{-\left\{(m-1) \lambda_{\mathrm{APH}}+n \lambda_{\mathrm{APF}}\right\} T_{2}}\right)$. (b) The probability that the HDN initiates the transmission and the AP has at least one packet for the nodes that are exposed to the HDN, which is calculated as $\frac{m \lambda_{\mathrm{H}}}{\lambda_{\text {Total }}}(1-$ $\left.e^{-\left\{(m-1) \lambda_{\mathrm{APH}}+n \lambda_{\mathrm{APF}}\right\} T_{2}}\right)(1-\gamma)$.

By adding (a) and (b), we can calculate the probability of HD communications occurring in this case.

Lemma 8 After initiating the transmission by an FDN, $H D$ communications will occur if (i) The AP has no data to send to any other node including the corresponding FDN or (ii) The AP has data to send to another node that is exposed to the corresponding FDN. SDTC-3 cannot be satisfied so $H D$ communications will occur in this case.

Proof To calculate the probability of HD communications occurring in this case, we need to calculate the following probabilities. (a) The conditional probability that the FDN initiates the transmission and the probability that the AP has no data to send to other nodes, including the FDN, within time $T_{1}$, which is calculated as $\frac{n \lambda_{\mathrm{F}}}{\lambda_{\text {Total }}}\left(e^{-\lambda_{\mathrm{AP}} T_{1}}\right)$. (b) The probability that after initiating the transmission by a FDN, the AP has no data for the corresponding FDN and the AP has at least one packet for the nodes that are exposed to the FDN within time $T_{1}$, which is calculated as $\frac{n \lambda_{\mathrm{F}}}{\lambda_{\text {Total }}}\left(e^{-\lambda_{\mathrm{APF}} T_{1}}\right)(1-$ $e^{-\left\{m \lambda_{\mathrm{APH}}+(n-1) \lambda_{\mathrm{APF}}\right\} T_{1}}(1-\gamma)$. By adding (a) and (b), we can calculate the probability of HD communications occurring when an FDN initiates the transmission.

Lemma 9 After initiating the transmission by the AP to an HDN, HD communications will occur if other nodes that cannot hear the RTS have no data for AP.

Proof To calculate the probability of HD communications occurring in this case, we need to calculate the following two probabilities. (a) The conditional probability that the AP has a packet to send to an HDN, which is $\frac{m \lambda_{\text {APH }}}{\lambda_{\text {Total }}}$. (b) The probability that other nodes that satisfy SDTC-1 have no data for the AP within time $T_{3}$, which is calculated as $\left(e^{-\left\{(m-1) \lambda_{\mathrm{H}}+n \lambda_{\mathrm{F}}\right\} \gamma T_{3}}\right)$. By multiplying (a) and (b), we obtain the probability of HD communications occurring in this case.

Lemma 10 After initiating the transmission by the AP to an FDN, HD communications will occur if (i) the corresponding FDN has no data for the AP and (ii) other nodes that cannot hear the RTSD have no data for the AP.

Proof To calculate the probability of occurring HD in this case, we need to calculate the following three probabilities. (a) The conditional probability that the AP has a packet to send to an FDN, which is $\frac{n \lambda_{\mathrm{APF}}}{\lambda_{\text {Total }}}$. (b) The probability that the corresponding FDN has no data to send to the AP within time $T_{1}$, which is $\left(e^{-\lambda_{\mathrm{F}} T_{1}}\right)$. (c) The probability that other nodes that cannot hear the RTSD have no data for the AP within time $T_{4}$, which can be written as $\left(e^{-\left\{m \lambda_{\mathrm{H}}+(n-1) \lambda_{\mathrm{F}}\right\} \gamma T_{4}}\right)$. By multiplying (a), (b), and (c), we can calculate the probability of HD communications occurring in this case.

By using Lemmas 7-10, the equation for calculating the probability of HD communication occurring $\left(P_{\mathrm{HD}}\right)$ can be derived as follows.

$$
\begin{aligned}
P_{\mathrm{HD}}= & \frac{m \lambda_{\mathrm{H}}}{\lambda_{\text {Total }}}\left(e^{-\left\{(m-1) \lambda_{\mathrm{APH}}+n \lambda_{\mathrm{APF}}\right\} T_{2}}\right) \\
& +\frac{m \lambda_{\mathrm{H}}}{\lambda_{\text {Total }}}\left(1-e^{-\left\{(m-1) \lambda_{\mathrm{APH}}+n \lambda_{\mathrm{APF}}\right\} T_{2}}\right)(1-\gamma) \\
& +\frac{n \lambda_{\mathrm{F}}}{\lambda_{\text {Total }}}\left(e^{-\lambda_{\mathrm{AP}} T_{1}}\right) \\
& +\frac{n \lambda_{\mathrm{F}}}{\lambda_{\text {Total }}}\left(e^{-\lambda_{\mathrm{APF}} T_{1}}\right)\left(1-e^{-\left\{m \lambda_{\mathrm{APH}}+(n-1) \lambda_{\mathrm{APF}}\right\} T_{1}}\right)(1-\gamma) \\
& +\frac{m \lambda_{\mathrm{APH}}}{\lambda_{\text {Total }}}\left(e^{-\left\{(m-1) \lambda_{\mathrm{H}}+n \lambda_{\mathrm{F}}\right\} \gamma T_{3}}\right) \\
& +\frac{n \lambda_{\mathrm{APF}}}{\lambda_{\text {Total }}}\left(e^{-\lambda_{\mathrm{F}} T_{1}}\right)\left(e^{-\left\{m \lambda_{\mathrm{H}}+(n-1) \lambda_{\mathrm{F}}\right\} \gamma T_{4}}\right)
\end{aligned}
$$

Furthermore, the probability equations should satisfy the following condition:

$$
P_{\mathrm{BFD}}+P_{\mathrm{TNFD}}+P_{\mathrm{HD}}=1
$$

\section{Result and performance analysis}

Simulations were performed in MATLAB to analyze the performance of the proposed HFD-MAC. The performance analysis for HFD-MAC is presented in the following two subsections in terms of the probability analysis and throughput analysis.

\subsection{Probability analysis}

The probability analysis was performed in unsaturated conditions, where every node did not have data to send at all times. By contrast, in saturation conditions, all clients and the AP always have packets to transmit [24]. The saturation condition represents the maximum load in a stable condition, i.e., the queue for arriving packets is assumed to always be nonempty for each node in the network.

The probability analysis results for BFD, TNFD, and HD transmissions in the proposed HFD-MAC relative to the 
downlink PAR (from AP to nodes) are shown in Fig. 9. The PGRs by each HDN and FDN were 20 and 25 packets/s, respectively, and the numbers of HDNs and FDNs were both 10 (fixed). The PGR was higher for FDNs than HDNs because FDNs can handle more data than HDNs. The downlink PAR at AP for each node was varied from 5 to 65 packets/s, as shown in Fig. 9. The packet arrival rate cannot be increased more than 65 packets/s for this simulation parameters because the utilization factor becomes higher than $100 \%$, which is a unstable condition. The maximum utilization factor in this case was about $94 \%$, i.e., the utilization factor was about $94 \%$ when the average PAR at AP for each node was 65 packets/s.

The simulation results in Fig. 9 show that the probability of HD communications decreased significantly as PAR increased at AP for each node, whereas $P_{\text {TNFD }}$ increased significantly as PAR increased for each node. Moreover, $P_{\mathrm{BFD}}$ also increased. Thus, the total probability of FD communications $\left(P_{\mathrm{TNFD}}+P_{\mathrm{BFD}}\right)$ increased significantly. Moreover, the figures present the effects of HTs on the probability of different communications. As shown in the figures, it is observed that the higher percentage of HTs in the network had higher probability of FD communications and lower probability of HD communications as compared between two sub-figures in Fig. 9. However, even with a small percentage of HTs (10\% of total nodes), the HFD-MAC provided about 70\% FD communications out of all possible communications in saturation conditions (Fig. 9a).

The probability analysis results according to the total number of nodes are shown in Fig. 10, where the PGRs and PARs were the same as those used in the previous analysis, i.e., 20 and 25 packets/s for HDNs and FDNs, respectively. In this simulation, $50 \%$ of total nodes were FDNs and $50 \%$ were HDNs always. The results in this figure exhibit a similar trend to those in the previous figure. The total probability of FD communications $\left(P_{\mathrm{TNFD}}+P_{\mathrm{BFD}}\right)$ increased greatly as the number of nodes increased whereas the probability of HD communications decreased. The maximum utilization factor in this case was about $97 \%$, i.e., the utilization factor was about $97 \%$ if the total number of nodes was 40. The results in Fig. 10 also depicts the effects of HTs on the probability of different types of communications in HFD-MAC. From this figure, it is observed that the probability of TNFD decreased with the increase of nodes' number, when the nodes' number was higher than 36 (Fig. 10b). $P_{\mathrm{HD}}$ continued to decrease and $P_{\mathrm{BFD}}$ continued to increase.

Based on this probability analysis, it is observed that the probability of FD communications $\left(P_{\mathrm{TNFD}}\right.$ and $\left.P_{\mathrm{BFD}}\right)$ increased significantly and the probability of HD communications decreased substantially as the system moved toward the saturation condition from an unsaturated condition. FD communications could not always be performed under saturation conditions because the SDTC was not always satisfied when HDNs initiated the transmission. In addition, the figures show that the sum of all the probabilities $\left(P_{\mathrm{TNFD}}, P_{\mathrm{BFD}}\right.$, and $\left.P_{\mathrm{HD}}\right)$ always equals 1 , thereby validating the derivation of the equations.

\subsection{Throughput analysis}

An extensive simulation was performed in MATLAB to analyze the throughput of our proposed HFD-MAC under saturation conditions. The simulation parameters are given in Table 4. The simulation results are shown in Fig. 12. HFD-MAC was compared with an existing FDMAC [17] and the traditional HD with RTS/CTS. We compared HFD-MAC with the existing FD-MAC because it also works in Het-WLANs. However, FD communications were limited only to cases where the AP and other FDNs had packets for each other [17].

We assumed that the reception of any control frame and data is successful if the receiving node lies within the range of the transmitter. A typical topology for an AP-based WLAN was considered for this simulation, as shown in Fig. 11. As mentioned earlier, satisfying the
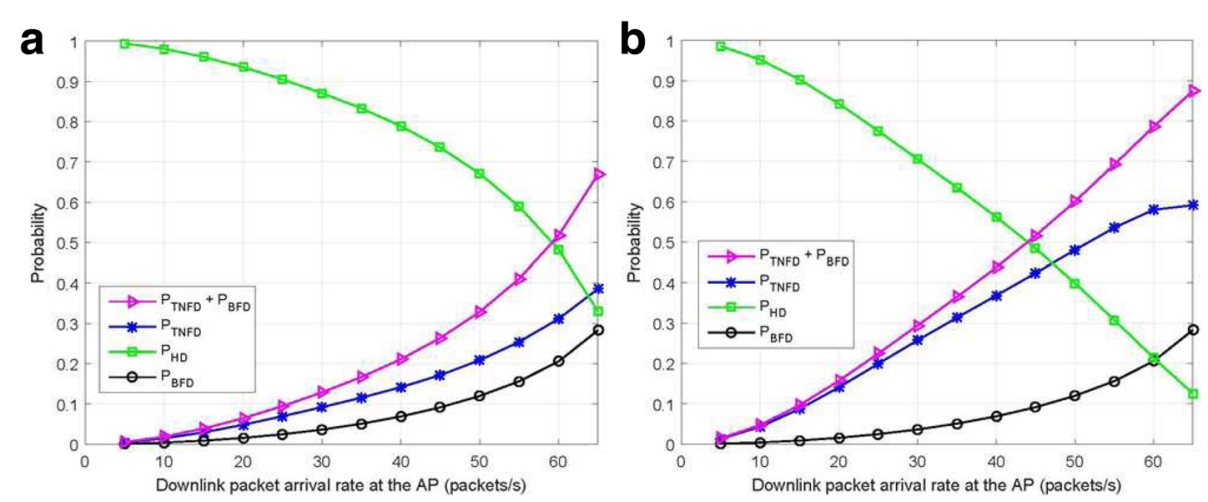

Fig. 9 Probability vs. downlink packet arrival rate at the AP. a Average HT ratio is 10\%. b Average HT ratio is 30\% 

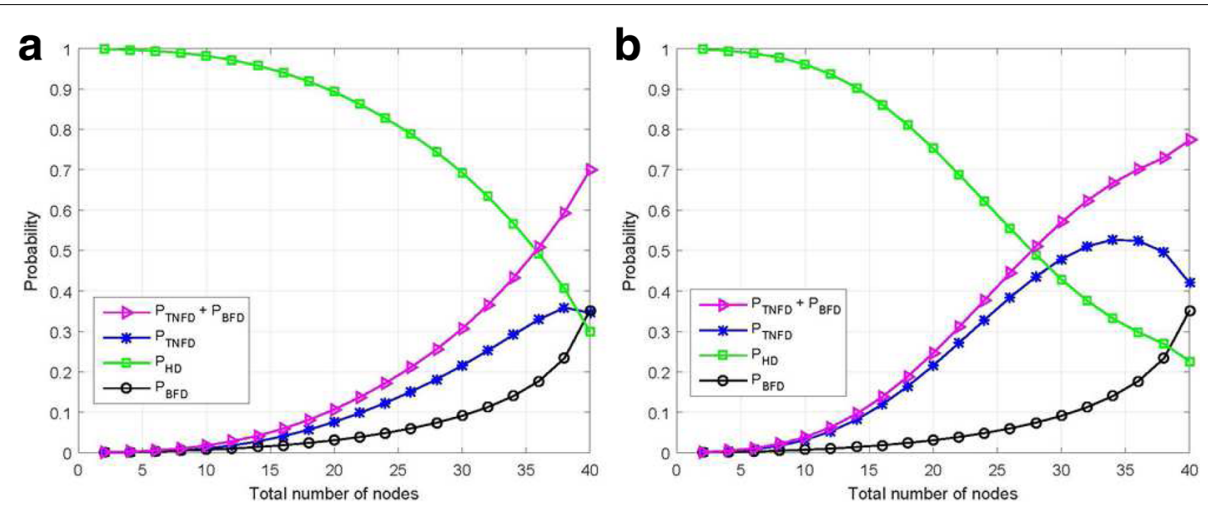

Fig. 10 Probability vs. total number of nodes. a Average HT ratio is $10 \%$. b Average $\mathrm{HT}$ ratio is $30 \%$

SDTCs is crucial for performing TNFD communications in HFD-MAC. Moreover, satisfying SDTC depends on the topology, where some hidden nodes exist. A WLAN was considered in this simulation with $30 \%$ hidden nodes on an average. For example, as shown in Fig. 11, if node B is a PT, then node $\mathrm{E}$ and $\mathrm{D}$ cannot hear the signal transmitted from B, i.e., E and D are HTs with respect to node B. Similarly, node F is the HT with respect to node C. Therefore, if $\mathrm{B}$ initiates the transmissions, E or D can participate in TNFD communications because they satisfy the SDTC. Similarly, if $C$ initiates the transmissions, only $F$ can participate in the TNFD communications.

As shown the Fig. 12, there were two FDNs and HDNs initially, and each number was then increased by two, whereas only one AP was considered. In this simulation, we used 10 samples as the throughput with each different number of nodes and then calculated the

Table 4 Simulation parameter

\begin{tabular}{ll}
\hline Packet payload (data) & 2000 bytes \\
RTS & 20 bytes \\
CTS & 14 bytes \\
RTSD & 20.25 bytes \\
CTSD & 14.25 bytes \\
ACK & 14 bytes \\
NDI & 14.25 bytes \\
Data rate & $54 \mathrm{Mbps}$ \\
Control frame (RTS, CTS, etc.) rate & $6 \mathrm{Mbps}$ \\
DIFS time & $34 \mu \mathrm{s}$ \\
SIFS time & $16 \mu \mathrm{s}$ \\
Slot time & $9 \mu \mathrm{s}$ \\
Minimum backoff window size $\left(C W_{\text {min }}\right)$ & 15 \\
PLCP preamble duration & $16 \mu \mathrm{s}$ \\
PLCP header duration & $4 \mu \mathrm{s}$ \\
Maximum time of self-timer & $50 \mu \mathrm{s}$ \\
\hline
\end{tabular}

average throughput, as shown in Fig. 12. The results show that the mean average throughput with HFD-MAC was $55.17 \mathrm{Mbps}$, whereas the values were 28.12 and 49.69 Mbps for the traditional HD and existing FD-MAC [17], respectively. Thus, the mean average throughput increased by 96.19 and $11.03 \%$ using our proposed MAC compared with the traditional HD and existing FD-MAC, respectively.

In this simulation, we considered that about $30 \%$ of total nodes were hidden when a particular node was considered. Satisfying the SDTC depends on the number of hidden nodes in the network. The throughput with HFDMAC was lower than that using the existing FD-MAC when the number of nodes was less than 10 because the SDTC cannot always be satisfied if the number of nodes

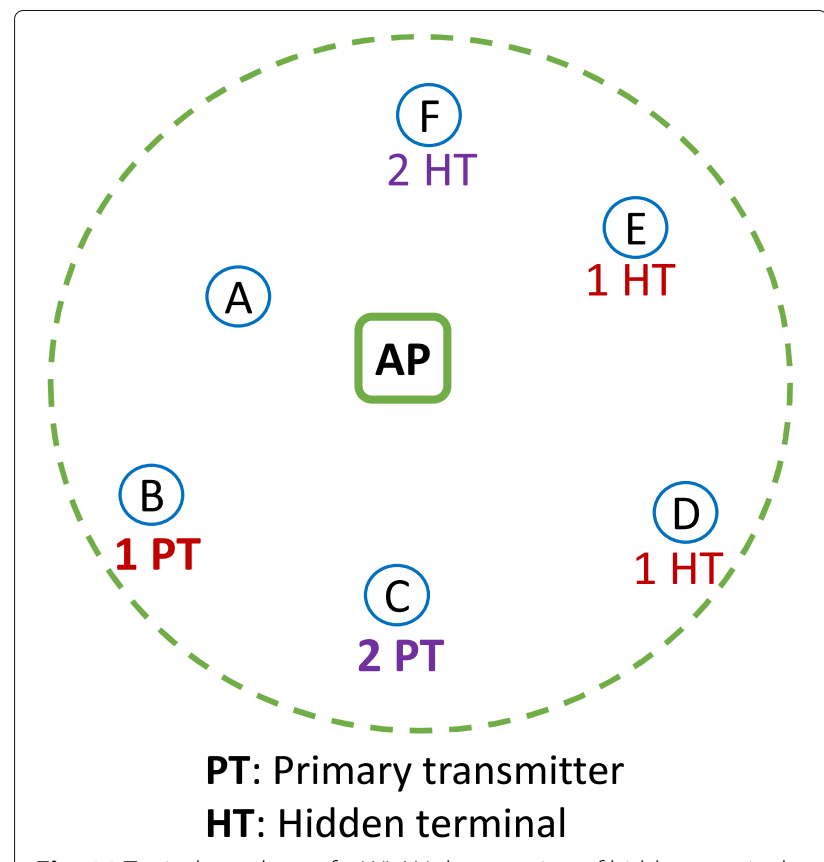

Fig. 11 Typical topology of a WLAN that consists of hidden terminals 


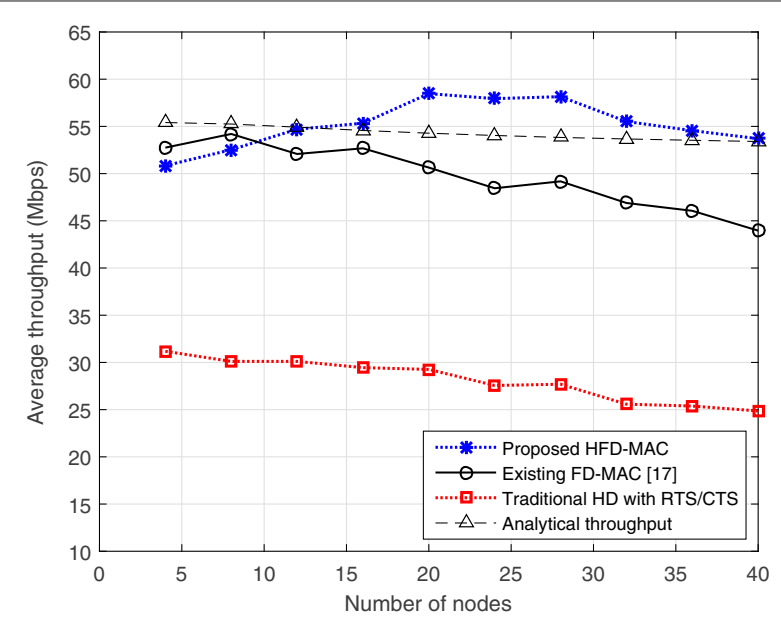

Fig. 12 Average throughput with respect to number of nodes

is lower. However, this SDTC condition is not required by the FD-MAC proposed by [17]. Hence, either BFD or HD communication occurred when using FD-MAC. Therefore, the average throughput was higher using FDMAC than the proposed HFD-MAC when the number of nodes was less than 10. In addition, the average throughput decreased with FD-MAC [17] when the number of nodes increased. When the number of nodes increased, BFD communications did not increase as much compared with TNFD communications. Therefore, the average throughput decreased with FD-MAC ([17]) because TNFD communications were not considered here. However, the probability of HD communications decreased greatly whereas that of TNFD increased significantly as the number of nodes increased, and thus, the average throughput increased in our proposed HFD-MAC.

Some differences are observed between the simulation throughput and the throughput that is calculated analytically as in Fig. 12. The reason is that the percentage of hidden terminals are fixed in the mathematical derivations. However, there are some variations of this number in different network topology in the simulation. Moreover, the contention period for channel access may vary in simulations, and thus, the exact time of the transmission also changes. On the other hand, a fixed average transmission time is assumed in the mathematical analysis. Therefore, some variations are observed between the simulation throughput and the analytical throughput. The mean average throughput is achieved by the simulation and the mean analytical throughput are 55.17 and 54.29 Mbps, respectively, which are very close to each other.

The average HT ratio is considered as $30 \%$ for the simulation in Fig. 12. This consideration is clarified by the following discussion. The MAC design is proposed for an infrastructure-based WLAN by using the game theory [25]. The authors evaluated the performance in the presence of HT and consider the percentage of HT from 10 to $60 \%$ [25]. The performance of IEEE 802.11 DCF is analyzed in the presence of HT, where the HT ratio is varied from about 5 to $60 \%$ [26]. The effect of HT on the performance of MAC protocol based on IEEE 802.11 standard are analyzed in [27]. The authors considered the HT ratio from 10 to $30 \%$ in that paper. The average HT ratio in practical radio environment can vary depending on various parameters, such as network topology, line of sight, and transmitting power or range. Therefore, we chose the average HT ratio as 30\% for this simulation in Fig. 12.

The performance of HFD-MAC in terms of average HT ratio for 25 nodes is shown in Fig. 13, which is performed in saturation conditions. As shown in the figure, the throughput of HFD-MAC increases as the average HT ratio increases. The SDTC conditions cannot be satisfied in most of the cases for the lower average HT ratio. However, the SDTC conditions are satisfied in most of the cases for higher average HT ratio and thus throughput increases. On the other hand, the throughput of existing FD-MAC [17] remains the same with the increase of average HT ratio, as there is no effect of HT on the throughput for that MAC protocol.

Further results are shown in Fig. 14 with 20 nodes, which compares the achievable maximum, average, and minimum throughput under saturation conditions. It is observed that the maximum achievable throughputs using our proposed HFD-MAC and the existing FD-MAC were almost the same at about $63 \mathrm{Mbps}$, whereas the value was $32.61 \mathrm{Mbps}$ with traditional HD. Hence, the maximum achievable throughput gain using our HFD-MAC was about $93.19 \%$ compared with HD communication. However, the gains in the average throughput were 96 and

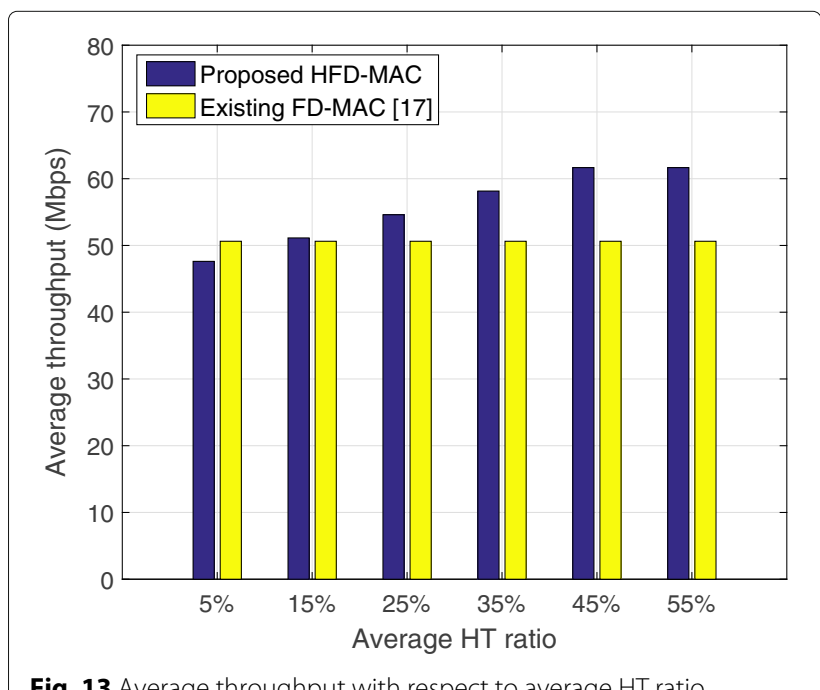

Fig. 13 Average throughput with respect to average HT ratio 


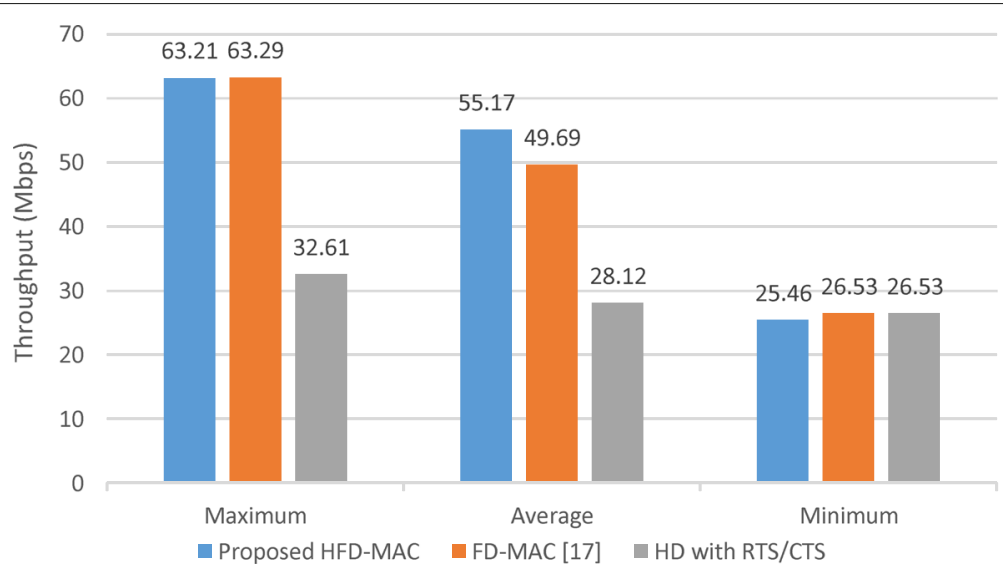

Fig. 14 Throughput comparison for 20 nodes

$11 \%$ comparing to the traditional HD and FD-MAC [17], respectively. On the other hand, HFD-MAC achieved the lowest throughput in case of the minimum throughput comparison. This happened in some rare cases in saturation conditions, when the TNFD was initiated only by a HDN and the SDTC was not satisfied, and thus, HD communications occurred. However in all other cases of TNFD, BFD communications occurred in saturation conditions. Moreover, as FD communications (either BFD or TNFD) occurred in most of times in saturation conditions, higher throughput was achieved by using the proposed HFD-MAC.

The MAC design for this kind of Het-WLAN is very important, as the coexistence of HDNs and FDNs in the same WLAN will be inevitable in near future. Some FDMAC were proposed, where FDNs can switch between FD and HD mode $[14,15]$. However, this is not sufficient because there are some differences in terms of MAC protocols between the WLAN that consists of only FDNs and the WLAN that contains both HDNs and FDNs. Although the FDN can switch between FD and HD mode, it depends on the packet availability and also on the response of the respective FDN (type 1). However, it is fixed that the HDN always performs HD transmission, which does not depend on packets arrival rate (type 2). The decision in type 1 is taken by the node and the AP acts accordingly. However, the decision in type 2 is always fixed, and the AP takes decision without getting any feedback from the HDN. Therefore, different MAC protocols are required for these two kinds of networks.

\section{Conclusions}

In this paper, we proposed an FD-MAC protocol for the Het-WLAN, which comprises FDNs, HDNs, and an FD-AP. In our proposed HFD-MAC, all possible FD transmissions were considered in Het-WLANs. This protocol minimizes the inter-user interference during FD transmissions by using SDTCs. The simulation results showed that HFD-MAC increased the overall throughput significantly compared with that using traditional HD transmissions. In addition, the performance of HFD-MAC protocol was better than that of another existing FDMAC. The probability analysis results suggested that the total probability of IBFD transmission increased significantly as the WLAN approached saturation conditions. This type of FD-MAC may be very important for supporting high-speed FD-WLANs in the near future.

\section{Acknowledgements}

This research and development work was supported by the MIC/SCOPE \# 155007006. This work was also supported by JSPS KAKENHI Grant Number JP16H01718.

\section{Competing interests}

The authors declare that they have no competing interests.

\section{Publisher's Note}

Springer Nature remains neutral with regard to jurisdictional claims in published maps and institutional affiliations.

Received: 1 July 2016 Accepted: 19 April 2017

Published online: 05 May 2017

\section{References}

1. D Bharadia, E McMilin, S Katti, Full duplex radios. ACM SIGCOMM Comput. Commun. Rev. 43(4), 375-386 (2013)

2. J Choi, M Jain, K Srinivasan, P Levis, S Katti, in Proc. of the Sixteenth Annual International Conference on Mobile Computing and Networking. Achieving single channel, full duplex wireless communication (ACM, Chicago, 2010), pp. 1-12

3. D Bharadia, S Katti, in Proc. of 11th USENIX Symposium on Networked Systems Design and Implementation (NSDI 14). Full duplex MIMO radios, (Seattle, 2014), pp. 359-372

4. M Jain, JI Choi, T Kim, D Bharadia, S Seth, K Srinivasan, P Levis, S Katti, P Sinha, in Proc. of the 17th Annual International Conference on Mobile Computing and Networking (MobiCom). Practical, real-time, full duplex wireless (ACM, Las Vegas, 2011), pp. 301-312

5. D Kim, H Lee, D Hong, A survey of in-band full-duplex transmission: from the perspective of PHY and MAC layers. IEEE Commun. Surv. Tutorials. 17(4), 2017-2046 (2015) 
6. KM Thilina, H Tabassum, E Hossain, DI Kim, Medium access control design for full duplex wireless systems: challenges and approaches. IEEE Commun. Mag. 53(5), 112-120 (2015)

7. Literature on asymmetry of the working document, SE19(13)49 Annex 5, 2013 (2013). http://cept.org/documents/se-19/10171/se19

8. E Everett, M Duarte, C Dick, A Sabharwal, in Proc. of the Forty Fifth Asilomar Conference on Signals, Systems and Computers (ASILOMAR). Empowering full-duplex wireless communication by exploiting directional diversity (IEEE, Pacific Grove, 2011), pp. 2002-2006

9. M Duarte, A Sabharwal, in Proc. of the Forty Fourth Asilomar Conference on Signals, Systems and Computers. Full-duplex wireless communications using off-the-shelf radios: feasibility and first results (IEEE, Pacific Grove, 2010), pp. 1558-1562

10. A Tang, X Wang, Balanced RF-circuit based self-interference cancellation for full duplex communications. Ad Hoc Netw. 24, 214-227 (2015)

11. M Sakai, H Lin, K Yamashita, in Proc. of IEEE Global Communications Conference (GLOBECOM). Adaptive cancellation of self-interference in full-duplex wireless with transmitter IQ imbalance (IEEE, Austin, 2014), pp. 3220-3224

12. Y Zhang, L Lazos, K Chen, B Hu, S Shivaramaiah, in Proc. of IEEE INFOCOM Conference on Computer Communications. FD-MMAC: combating multi-channel hidden and exposed terminals using a single transceiver (IEEE, Toronto, 2014), pp. 2742-2750

13. W Cheng, X Zhang, H Zhang, in Proc. of IEEE Global Communications Conference (GLOBECOM). RTS/FCTS mechanism based full-duplex MAC protocol for wireless networks (IEEE, Atlanta, 2013), pp. 5017-5022

14. S Goyal, P Liu, O Gurbuz, E Erkip, S Panwar, in Proc. of Asilomar Conference on Signals, Systems and Computers. A distributed MAC protocol for full duplex radio (IEEE, Pacific Grove, 2013), pp. 788-792

15. A Sahai, G Patel, A Sabharwal, arXiv preprint arXiv:1 107.0607 (2011)

16. W Choi, H Lim, A Sabharwal, Power-controlled medium access control protocol for full-duplex wifi networks. IEEE Trans. Wirel. Commun. 14(7), 3601-3613 (2015)

17. M Duarte, A Sabharwal, V Aggarwal, R Jana, K Ramakrishnan, CW Rice, N Shankaranarayanan, Design and characterization of a full-duplex multiantenna system for wifi networks. IEEE Trans. Veh. Technol. 63(3), 1160-1177 (2014)

18. A Tang, X Wang, A-duplex: medium access control for efficient coexistence between full-duplex and half-duplex communications. IEEE Trans. Wirel. Commun. 14(10), 5871-5885 (2015)

19. JY Kim, O Mashayekhi, H Qu, M Kazandjieva, P Levis, Janus: A novel MAC protocol for full duplex radio. CSTR. 2(7), 23 (2013)

20. T Riihonen, S Werner, R Wichman, E Zacarias, in Proc. of the 10th Workshop on Signal Processing Advances in Wireless Communications. On the feasibility of full-duplex relaying in the presence of loop interference (IEEE, Perugia, 2009), pp. 275-279

21. W Cheng, X Zhang, H Zhang, in Proc. of IEEE INFOCOM Conference. Full/half duplex based resource allocations for statistical quality of service provisioning in wireless relay networks (IEEE, Shanghai, 2012), pp. 864-872

22. T Riihonen, S Werner, R Wichman, Hybrid full-duplex/half-duplex relaying with transmit power adaptation. IEEE Trans. Wirel. Commun. 10(9), 3074-3085 (2011)

23. IEEE Standards Association, 802.11-2012-IEEE standard for information technology - telecommunications and information exchange between systems local and metropolitan area networks — specific requirements part 11: wireless lan medium access control (MAC) and physical layer (PHY) specifications (2012). http://standards.ieee.org/about/get/802/802. 11.html

24. G Bianchi, Performance analysis of the IEEE 802.11 distributed coordination function. IEEE J. Sel. Areas Commun. 18(3), 535-547 (2000)

25. SM Najafabadi, CC Constantinou, in Proc. of IEEE 24th International Symposium on Personal Indoor and Mobile Radio Communications (PIMRC). A game theoretic model for wireless medium access control in the presence of hidden terminals (IEEE, London, 2013), pp. 1731-1736

26. H Wu, F Zhu, Q Zhang, Z Niu, in in Proc. of IEEE Global Telecommunications Conference. WSNO2-1: analysis of IEEE 802.11 DCF with hidden terminals (IEEE, San Francisco, 2006), pp. 1-5

27. S Khurana, A Kahol, AP Jayasumana, in Proc. in IEEE Conference on Local Computer Networks (LCN'98). Effect of hidden terminals on the performance of IEEE 802.11 MAC protocol (IEEE, Lowell, 1998), pp. 12-20

\section{Submit your manuscript to a SpringerOpen ${ }^{\circ}$ journal and benefit from:}

- Convenient online submission

- Rigorous peer review

- Immediate publication on acceptance

- Open access: articles freely available online

- High visibility within the field

- Retaining the copyright to your article

Submit your next manuscript at $\boldsymbol{\triangleright}$ springeropen.com 